\title{
Genese, developpement, status et futur du projet d'harmonisation des principes et normes transnationales de la procedure civile transnationale $\left({ }^{*}\right)\left({ }^{* *}\right)$
}

\section{Aníbal Quiroga León}

\begin{abstract}
A Mme. Frédérique Mestre, juriste française, avec l'amitié et reconaissance. Et ainsi à ma colegue et amie Maître Ana Djoric, de

Serbie, et a tous les fonctionnaires, employés, collaborateurs et boursiers de l'UNIDROIT qui, d'une ou autre manière, sans savoir,

font partie de ce texte.
\end{abstract}

(*) Rapport final sur l'investigation réalisée sous les auspices de la bourse octroyée par le programme de bourses de l'UNIDROIT, Rome, Italia, du 01 avril-02 au 02 juillet 2002 ; et sous les auspices du Maître Héctor Fix-Zamudio, Chercheur Emérite de l'Institut de Recherche Juridiques de l'UNAM, Mexique; Ex Président de la Cour Interaméricaine de Droits de l'Homme, Docteur Honoris Causa par l'Université Catholique Pontificale du Pérou, Président de l'Institur Ibéro-américain de Droit Constitutionnel, Membre de I'Institut International de Droit Processuel; de l'Institut de Droit Agricole International et Comparé; de l'Institut Espagnol de Droit Processuel; de l'Institut International des Droits Humains de Strasbourg et de l' Académie Mexicaine de Législation et Jurisprudence ; et par le Maître Domingo García Belaunde, Professeur Principal de la Faculté de Droit de l'Université Catholique Pontificale du Pérou, Docteur de Droit par l'Université Nationnel Majeur de Saint Marcos, Lima, Pérou; Président de l'Association Péruvienne de Droit Constitutionnel. Membre de la Direction et fondateur de l' Institut Ibéro-Américain de Droit Constitutionnel. Membre de la Commission de Bases de la Réforme Constitutionnelle Péruvienne. Membre de l'Académie Péruvienne de Droit, de l'Association Argentine de Droit Constitutionnel, de l'Académic Nationale de Droit et Sciences Sociales de Córdoba, Argentine. Toutefois, les opinions et le contenu présentés dans ce travail restent la seule responsabilité de l'auteur et n'engagent pas d'aucune manière la responsabilité de l'Unidroit. Traduction en français a été faite par Mme. Hilda Asencio, de Lingue S.A., et revisé par le Prof. Ana Djoric, de l'Institut de Droit Comparé à Belgrade, Serbie ; et par l'auteur.

(**) La recherche a été réalise grâce à l'aide précieuse de Corporación Aceros Arequipa S.A., ainsi comme autres colaborations qui veunt rester dans l'anonimée; l'Entreprise et personnes à laquelles l'auteur est personnellement très reconaisant. 
«...un homme laissa tomber trois monnaies et un autre les trouva. Tous deux se présentèrent devant le juge et tous deux insistaient disant qu'aucun des deux n'avait le droit de conserver les monnaies. Celui qui laissa tomber les monnaies expliqua qu'il ne méritait pas de les garder car leur perte avait été causée par sa propre négligence. Celui qui les avait trouvées argumenta qu'il ne pouvait les garder car elles ne lui appartenaient pas [...] Après avoir écouté les parties, le juge sortit trois autres monnaies de sa propre poche, les ajouta aux autres et puis partagea les six en trois parties, une partie pour chacun... Et finalement il dit: celui qui a laissé tomber les monnaies a maintenant deux monnaies, celui qui a trouvé les trois monnaies n'en a maintenant plus que deux; j'avais moi-même trois monnaies dans ma poche et à présent je n'en ai plus que deux. Chacun de nous a perdu une monnaie. Ceci est ma sentence."

Decision judiciaire de la periode feodale EDO

$(1600-868)^{\prime}$

\section{Présentation}

On dit qu'être reconnaissant est le fait de personnes bien nées. C' est pourquoi je voudrais d'abord remercier l'UNIDROIT pour m'avoir octroyé la bourse de recherche qui m'a permis à réaliser la présente étude, et m'avoir donné la possibilité de me rendre à Rome pour trois mois afin de me'consacrer entièrement à ce travail académique. C'est pourquoi je tiens très particulièrement à remercier en premier lieu à I'UNIDROIT pour m'avoir offert la bourse de recherche qui m'a permis de travailler à Rome, en Italie, où se trouve son siège, et qui a rendu possible l'avancement et le développement de cette étude qui aujourd'hui, d'une manière préliminaire, permet la publication de ce travail. L'effort pour trouver des normes uniformes dans les procédés judiciaires de notre ère, et leur réponse actuelle dans le Projet de Principes et Normes ( ou règles) Transnationales du Procès Civil constituent-sans aucun doute- un espoir pour le présent et un défi pour le futur; défi pour être de pair avec l'inexorable processus de

1 Lorenzo Zolezzi - "Relevancia social del Juez Civil» - Communication, ILANUD, San José, 1987. 
mondialisation et l'unification progressive des Systèmes Juridiques; et l'espoir de doter les différents pays - et très souvent non plus si "différents»-c'est-à-dire, à la Communauté Internationale, de meilleures conditions et instruments pour l'obtention de la justice, de règles justes, uniformes et prévisibles pour les différentes administrations de Justice, de normes homologuées pour les différentes solutions de conflits individuels qui se présenteront toujours au sein d'une société, comme partie consubstantielle de l'être humain et de sa vie en groupe, et qu'en raison de l'intense échange commercial, économique, culturel et social, étend la genèse et la solution de ces conflits aux justiciables de l'une ou l'autre communauté nationale, de l'une ou de l'autre côté de la frontière, de l'une ou de l'autre côté des océans.

J'ai eu l'honneur d'être invité fin 1999 à joindre le Projet de Principes et Règles du Procès Civile Transnational, défi et illusion mêlés, qualifié par certains d'utopie ou d'irréalisable, ce qui, pour moi, s'est matérialisé comme Consultant International pour le Pérou dès mars 2000, grâce à l'aimable invitation que me formulèrent les professeurs Goeffrey C. Hazard Jr. Co-Rapporteur et Antonio Gidi, rapporteur associé, de l'American Law Institute'.

L'expérience universitaire et professionnelle des opérateurs juridiques et, spécialement, de ceux qui, comme moi-même, se consacrent à l'étude et à la pratique du litige civil et commercial, nous a permis de réfléchir à plus d'une occasion sur la nécessité d'uniformiser les règles qui pourraient être appliquées dans les controverses juridiques qui lient les entités privées et dont les effets se font sentir dans différents pays.

Aujourd'hui l'UNIDROIT, avec son développement institutionnel et son prestige international ${ }^{3}$, a rendu possible une période de recherche

2 Comme conséquence de cette coopération fructueuse, je fus invité en octobre 2000 à assister au Séminaire International "Vers un procès civil universel? Les règles transnationales de procédure civile de l'American Law Institute», organisé par l' Université Panthéon-Assas (Paris II) et l' American Law Institute, à Paris, où il a été publié le texte Vers un procès civil universel? Les règles transnationales de procédure civile de l' American Law Institute, édition effectuée sous la direction du Professeur Philippe Fouchard.

3 L' UNIDROIT est une organisation internationale intergouvernementale indépendante dont le siège est à Rome, créée en 1926 et reconstituée en 1940 sur les bases des Statuts de UNIDROIT, ayant comme le but principal la réflexion et l'étude des voies 
centrée précisément sur cette matière et qui s'est déroulée sous la conduite et avec la vision de Maître Frédérique Mestre, prestigieuse juriste française, qui a concrétisé cette possibilité ouvrant les portes de cette thématique à l'espace latino-américain.

Dans ce cas, le défi le plus important et aussi le grand écueil, consiste en devoir nécessairement établir des relations entre des institutions processuelles nées au sein des deux grands systèmes juridiques. C'està-dire ceux qui appartiennent au Common Law et au Civil Law. ${ }^{4}$

La dynamique actuelle du monde dans lequel nous vivons, l'universalisation vers laquelle se dirigent les nouveaux systèmes d'information, très rapides, et les nouvelles modalités d'engagement sous contrat dans un commerce international de plus en plus intense, et ses nouvelles formes économiques, constituent en soi des motifs plus que suffisants pour tenter d'obtenir, dans un délai raisonnable, l'unification de la procédure des controverses civiles et commerciales qui surgissent comme conséquence inévitable de cette mondialisation. Celle-ci se manifestera inexorablement en une globalisation du litige, de la controverse et de la crise des relations sociales et individuelles dans les différentes communautés nationales, en promouvant la nécessité de la création de solutions juridiques au niveau macro, extraterritoriales, nécessairement « universalisantes ». Ce Projet et ces efforts, dignes d'être tentés, s'inscrit précisément dans cette direction, sous l'axiome qui déclare que la foi est la source de la créativité humaine.

Il est impensable à l'heure actuelle que les frontières nationales continuent à être en même temps barrières culturelles, sociales ou économiques, comme ces murailles qui séparaient, pour leur défense et leur autarcie, les vieilles villes.

d'harmonisation du droit civil et commercial - dans diverses manifestations entre les États - sans exception, qu'ils soient ou non membres de l'UNIDROIT, comme c' est le cas pour le Pérou qui n'en fait pas encore partie- et entre les groupes d'États, ainsi que la préparation graduelle de matériaux et projets pour l'adoption de normes communes, principes et traités internationaux concernant cette matière.

4 Voir Anibal Quiroga Leon.- Présentation de l'Edition spéciale de DERECHO PUC, «Projet de Normes Transnationales du Procès Civil», Fonds Editorial PUCP du Pérou, Lima, déc. 1998-mars 1999. Egalement de : FOUCHARD, Philippe.- "Vers un procès civil universel ? Les règles transnationales de procédure civile de l' American Law Institute, Débats» : Anibal Quiroga León, pp. 29-30 Ed. Panthéon Assas, Paris 2000 
C' est pourquoi la finalité de cet important Projet, étant donné le développement et l'augmentation des transactions commerciales d'ordre international qui font de nécessité publique qu'on ne peut remettre à plus le fait d'avoir des mécanismes communs de sécurité et des formes de procédure en accord avec l'ampleur de leur développement, de manière telle que le système juridique fournisse aux justiciables des formules simplifiées, expéditives et communes, a pour la solution des controverses qui pourraient se soulever, avec la double finalité, et en paraphrasant l'insigne Maître uruguayen Eduardo J. Couture, de résoudre facilement, économiquement et rapidement les inévitables conflits particuliers, offrant en même temps la sécurité et la paix à la Communauté Internationale pour atteindre ce but.

Ceci, naturellement, avec un vrai respect des normes nationales et internationales qui garantissent un Procès légal en bonne et due forme, ou Tutelle Juridictionnelle Effective aux justiciables, précisément selon les standards internationaux que ce concept universalisant a développé jusqu'à présent. Pour ce faire, il est d'une importance transcendantale de compter sur une normativité uniforme et acceptée à l'échelle internationale qui permette que les systèmes de justice inter et intra-nationaux fonctionnent en accord avec ces circonstances, contribuant ainsi au développement économique et social des nations.

Pour atteindre ce résultat, il est indispensable le concours et l'aide de la Théorie Générale du Procès afin de systématiser, grouper par thèmes et expliquer les différents principes et les progrès, les sources mêmes des instruments processuels qui déterminent leur nécessité et la possibilité d'unification ou d'homologation. Sans craindre d'exagérer, on pourrait comparer la genèse et le développement qualitatif de ce projet avec l'histoire de la science du procès, lorsqu'en 1868, Oskar von Bülow publia l'oeuvre qui marqua la fin du "procéduralisme " et donna naissance à l'ère scientifique du procès avec l'Ecole Publiciste en Allemagne, prédécesseur de l'italienne et de l'espagnole ${ }^{5}$ C'est Ibañez $\mathrm{DE}$ Aldecoa ${ }^{6}$ qui a signalé de façon très intelligente que:

5 Oskar von Bülow.- "La théoriẹ des exceptions processuelles et les Budgets Processuels "; ( Giesen, 1868) traduction de Miguel Angel Rozas Lichtschein ed. Juridiques Europe Amérique, Buenos Aires 1964

6 Alfonso Ibañez de Aldeoca.- Meditaciones sobre la cientificidad dogmática del Derecho Procesal- Contraportada. 
"Le Droit processuel a le privilège d'avoir motivé, ces dernières années, l'inquiétude de la recherche, et les "processuelistes " se sont mobilisés pour l'accomplissement d'une tâche transcendante en assumant la responsabilité de construire scientifiquement leurs questions, de grouper par thèmes et "problematiser " leurs points vitaux qui restaient cachés dans les systèmes, de présenter le procès comme une institution qualifiée digne de recherche qui possède une force et une autonomie sur laquelle on pourra construire une spécialisation juridique avec des contours fermes et propres".

Jusqu'à présent, notre participation au présent projet, concernant de façon fondamentale sa diffusion, interprétation, explication et traduction à l'espagnol de ses différentes versions, spécifiquement en ce qui concerne différents termes légaux utilisés en Amérique latine mais qui très souvent signifient la même chose ${ }^{7}$, n'a pas été tâche facile. Non seulement parce que dans la doctrine du droit, et dans la Théorie du Droit également sans doute, il existe des concepts synonymes, des institutions similaires et des usages locaux, très souvent notre propre formation professionnelle et juridique nous mène à penser que notre signification est la seule pertinente, ou que nos localismes doivent s'imposer, ou qu'accepter un autre implique une sorte de "dépersonnalisation " juridico-judiciaire.

Rien de plus opposé, précisément, à une théorie générale qui prétend être globalisante dans l'art du jugement humain, aussi commun que la vie, l'air, l' eau ou la vie en commun en démocratie.

Nous avons également participé, il n'y a pas longtemps et en collaboration avec l' American Law Institute, à la diffusion du dernier Projet de Normes Transnationales au moyen de la Revue DERECHO PUC No 52, organe officiel de diffusion universitaire de la Faculté de Droit de l' Université Catholique Pontificale du Pérou dont je suis l'Editeur Général. Dans un travail conjoint avec le Professeur Antonio Gidi et grâce à l'aide de l' American Law Institute nous avons réussi à éditer dans ce but une édition spéciale d'un tirage à part de notre numéro DERECHO PUC $52^{8}$ où sont incluses les versions en anglais, Espagnol et Portugais de la version anglaise du Discussion

7 Aníbal Quiroga León,.- Op. Cit.

8 Ibidem 
Draft du 1 avril 1999 du Projet, dans le but de pouvoir la diffuser dans les communautés hispanophones et lusophones, aussi bien de l'Amérique que de l'Europe et même de l'Afrique.

L'Amérique Latine a aujourd'hui un grand défi à relever : réussiren même temps que l'unification de ses marchés, de ses économies et de ses politiques- à unifier sa législation processuelle, sa justice, la vitale, que réclament ses populations; et ce projet a démarré avec l'élaboration du Code Processuel Civil Modèle pour l'Amérique Latine, et, actuellement, un projet similaire se prépare dans le domaine pénal. Il convient de signaler que nos racines législatives, l'unité idiomatique et surtout notre tradition juridique nous permettent de regretter la unification si souhaitée en matière processuelle? . Ce désir et ce défi lancés par les différentes institutions qui regroupent les chercheurs du Droit Processuel dans toutes ses branches, pourraient se voir renforcé par la diffusion des Principes et règles Transnationales du Procès Civil. La recherche de l'unification législative dans le domaine processuel n'est pas éloignée de la pensée latino-américaine et cela peut servir de base pour une plus grande diffusion de cet important projet.

Pourquoi penser à une législation processuelle transnationale unifiée en matière civile et commerciale? Cette interrogation n'est pas non plus éloignée de la réalité latino-américaine. Il suffit de voir pour s'en convaincre que la législation processuelle fédérale et provinciale argentines en matière civile et commerciales marchent de concert toujours unies. S'il est vrai que cet exemple n'a pas été adopté par d'autres pays de l'Amérique Latine, nous ne pouvons nier, nous qui exerçons dans le domaine du litige, que l'une et l'autre branche de la procédure sont presque toujours unies. Il est pratiquement impensable de croire que le procès civil puisse se séparer du procès commercial; pour s'en convaincre il suffit de voir que les affaires juridiques commerciales étudiées auparavant avec le Droit Civil sont de plus en plus influencées par le droit commercial et si nous allons plus loin, nous verrons comment le droit commercial est de plus en plus amalga-

9 C' est à l'auteur du présent exposé, qu'au cours du dernier VIle Congrès Iberoaméricain de Droit International, au mois de février, à Mexique D.F. sur la base de cette expérience accumulée, que lui a été faite la proposition singulière de mettre en train l'étude et la rédaction d'un projet de Code Processuel Constitutionnel Modèle pour Ibéroamérique. 
mé au droit international privé, car les transactions civiles commerciales qui, il y a 50 ans, se circonscrivaient uniquement dans le milieu local, peuvent aujourd'hui traverser les frontières en moins d'une minute par le biais de transactions électroniques ou digitales qui ont transformé le droit commercial en un droit d'ordre international. Ce progrès dans les transactions, aujourd'hui électroniques, requiert le développement (à court terme) d'un support processuel élémentaire et unifié où ne pourront pas prévaloir les régionalismes (voyez les lois de la Communauté Européenne où peu à peu ses lois remplacent les lois nationales des Etats membres) dans lequel on aborde la tâche difficile mais nécessaire d'unifier les règles processuelles qui devront être établies et prévaloir face à ces progrès "transnationaux" du droit civil et commercial.

En résumé, les conditions sont données pour pouvoir développer, diffuser et étudier avec persévérance cet important Projet d'Unification des Règles (ou normes) Transnationales de la Procédure civile. L'Amérique Latine a l'expérience nécessaire au sujet de la préparation et de l'utilisation de projets modèles ou Codes Base, ainsi que l'avantage du fait de parler la même langue et d'avoir une seule tradition juridique, c'est-à-dire qu'elle a plus de facilités pour pouvoir prendre part à cet ambitieux projet et nous n'avons besoin que de la volonté et du travail pour la diffusion de ces normes. Cela nous permettra dans un futur qui, sans être proche, pourra cependant être atteint, d'obtenir tout d'abord l'unification des règles élémentaires qui doivent prévaloir dans toute procédure d'ordre civil et commercial, et plus tard nous incorporer pleinement à la régulation transnationale qui est ce que nous prétendons réussir avec ce projet.

\section{Sur la theorie generale du proces}

Le Droit Processuel a reçu, en général, une estimation secondaire au sein des différentes Ordres Juridiques; ce qui est encore pire, à la différence d'autres disciplines juridiques, souvent même on n'y a pas suivi les courants doctrinaires modernes ni connu les progrès scientifiques en matière de procédure.

C'est ainsi que c'est bien peu ce que l'on admet -et moins encore ce que l'on sait- sur la dogmatique juridique du procès et sa Théorie Générale, ses institutions et la qualité scientifique de ses postulants, 
telle qu'elle est reconnue aujourd'hui, et surtout sur l'importance capitale qu'elle a pour l'Ordre Juridique, puisqu'elle dépasse sa propre limite institutionnelle pour atteindre des terrains plus lointains, y compris les constitutionnels.

Si nous admettons que le Droit n'est pas un simple système de règles statiques et saisissables réglant la société au moyen des normes toujours en vigueur, soit volontairement ou de façon coercitive, avec la médiation de l'Organe Juridictionnel, mais que par contre, comme l'affirme Latorre ${ }^{10}$ c'est une science d'existence sociale dont les règles juridiques doivent leur complexité à la nature même de la société, nous comprendrons que le développement atrophié de l'un de ses piliers sera un obstacle pour empêcher la consécution de la justice comme fin indirecte et la paix sociale avec la solution des conflits intersubjectifs comme fin immédiate.

C'est que la justice, au dire de Devis Echeandia ${ }^{11}$, comme notion abstraite, est invariable; mais la manière de l'obtenir dans la pratique, difficile et rébarbative, est nécessairement changeante parce qu'elle doit s'adapter à la évolution permanente du milieu social et de la personne humaine elle-même. Chaque période historique a eu un concept différent de la justice et un procédé spécial pour l'adopter, sans qu'auparavant ni à présent on n'ait pu atteindre la perfection et peut-être ne l'atteindronsnous jamais, ni même cette justice relative et incomplète dont s'accommode souvent l'homme et qui est la seule à sa portée.

C'est la raison d'être du Droit Processuel et le fondement de la procédure. C'est pour cela que seulement au moyen de son développement adéquat puissent se profiler les relations de justices adéquates et ce n'est que par cela que l'on peut prétendre à un développement social et humain cohérent. C'est pourquoi, en partant dès le début, il faut reconnaître que l'ordre juridique nécessite une régulation processuelle adéquate et que cette régulation s'adresse, à son tour, à toutes les branches du droit. Il est illusoire de prétendre au développement d'une science juridique nationale sans le progrès coordonné et réciproque de toutes les institutions qui l'en sont les

10 Angel Latorre.- Introducción al Derecho, Ed.Ariel, Barcelona, 1969, pages 80-

11 Hernando Devis Echandia.-"IV Jornadas Latinoamericanas de Derecho Procesal" dans Revista de derecho español y Americano No 17 
piliers. Le Droit Processuel est un soutien important pour la réalité en vigueur et le caractère effectif des relations subjectives qui règlent la vie sociale et la relation entre l'État et les justiciables.

L'importance du procès apparaît clairement dans ses principaux fondamentaux: la soustraction à l'homme de la possibilité de donner une solution privative à ses conflits particuliers. A ce sujet, Aragones ${ }^{12}$ soutient qu'en son absence, l'homme associerait ses origines à ce que l'autotutelle ou autodéfense définissait comme le premier élan du sentiment du droit contre l'injustice: l'action violente, l'imposition de la force avant la raison, l'origine de la défense personnelle et la vengeance, cette justice sauvage qui a disparu, précisément, avec l'existence de l'État de Droit.

Cela était mentionné dès les études préliminaires du Droit Processuel comme science tel que le déclare le Conde de la Cañada ${ }^{13}$ lorsqu'il dit que:

"Les hommes, qui dans leur état primitif naturel ne considéraient pas supérieur que l'on les défendais des insultes, des oppressions et des violences, étaient alors autorisés à se défendre eux-mêmes: l'expérience leur fit comprendre les graves dangers qu'ils encouraient car, ou ils ne pouvaient pas se défendre eux-mêmes, ou dépassant les justes limites pour se conserver, excitaient les troubles et provoquaient de plus grandes mésententes, des injures et des morts ; et consultant d'autres moyens qui pourraient améliorer la sécurité de leurs personnes sans les risques mentionnés plus haut, ils décidèrent de s'unir en sociétés et confier leur défense et celle de tous leurs droits à une personne qui les regardant avec impartialité pourrait leur distribuer leurs droits et les conserver en justice et en paix.»

C'est pourquoi, comme manifeste Gonzalez Perez ${ }^{14}$, ce n'est qu'interdisant l'exercice de la force privée pour satisfaire les prétentions et la reconnaissance des droits que l'on pourra assurer le règne de la

12 Pedro Aragones Alonso.- Proceso y Derecho Procesal; Aguilar; Madrid, 1960; p. 27

13 Cité par Jesús González Pérez.- El Derecho a la Tutela Jurisdiccional; Ed. Civitas, S.A.; Madrid, 1984, p. 19

14 Op. cit, p. 20 
justice. D'un chaos où dominait la loi du plus fort on est passé à l'ordre juridique où prévaut le critère d'un sujet impartial, en substituant l'action directe face à l'adversaire par l'action dirigée vers l'État, afin que les organes spécialement institués par lui accueillent et jugent les prétentions d'un sujet face à l'autre. L'histoire de la substitution de l'autotutelle ou l'autodéfense par le procès a été, en définitive, l'histoire du développement social de l'homme.

Mais il est nécessaire de tenir compte -pour éviter un développement conceptuel linéaire- du fait que si l'histoire de l'humanité n'a pas été une augmentation progressive et constante des vertus morales de l'homme elle n'a pas été non plus une réduction progressive de l'autodéfense au bénéfice du procès. Il y a eu des périodes pendant lesquelles tout s'est déroulé selon la loi et d'autres où sont apparues des manifestations notoires d'autodéfense qui paraissaient avoir été définitivement abandonnées. Ceci concerne particulièrement les avatars de notre monde contemporain et, plus récemment, notre société nationale sévèrement menacée par la violence. Dans notre monde actuel, la violence se présente en termes plus virulents et généralisés que jamais, et l'on peut même parler de l'actualité de la vengeance ${ }^{15}$ pour refléter la réapparition de l'autodéfense sous ses formes les plus rancunières et arbitraires. Quelles sont à vrai dire, les causes de cette réapparition défensive? Elles sont plusieurs et d'origines variées, dit Gonzales Perez ${ }^{16}$ mais peut-être - et peut-être sans peut-être - en premier lieu la méfiance, et même le mépris du citoyen pour la justice offerte par l'État. Les droits des citoyens, surtout les droits fondamentaux, se voient sévèrement transgressés lorsque le respect envers l'autorité de l'État en tant que dirimant des conflits sociaux et intersubjectifs est bafoué; aussi forts et même brutaux que puissent être les éléments coercitifs que l'État peut employer, ils seront débordés par la quête désespérée de justice. De là on peut se rendre compte de l'importance $\mathrm{du}$ procès comme instrument du procès juste ou une Tutelle Judiciaire Effective, définis comme la possibilité pour personne ayant un droit en dispute puisse se présenter devant l'Organe Juridictionnel afin que sa controverse soit résolue convenablement, c'est-à-dire en

15 Titre du livre de Ruiz Funez, Bs As, 1044 ;Cit.par Alcala Zamora et Niceto CastiIlo.- Proceso, autocomposición y Autodefensa, México, p.64, p.21 et Cit. 4

16 Jesus Gonzalez Perez.-Op.cit. p. 21 
rendant effectif le droit matériel du cas concret et faisant à son tour réalité l'idéal de justice inhérent à ce droit applicable à ce cas concret.

A partir de cela, on peut déduire qu'une régulation processuelle défectueuse interférera au- delà de l'aspect purement juridique jusqu'à arriver aux propres relations sociales, comme le signale l'auteur italien Piero Calamandrei ${ }^{17}$ en déterminant que la norme juridique processuelle est la règle technique sous tutelle de l'État et investie d'autorité avec laquelle on accède à la vie pacifique de la société et qui constitue l'exercice de la fonction la plus solennelle et plus élevée de l'État : la justice, qui est le fundamentum republicae.

C'est ce qu'un autre grand auteur italien, Mauro Cappeletti ${ }^{18}$ explique lorsqu'il signale que le Droit Processuel est vu comme un phénomène lié aux commotions extraordinaires souffertes par l'homme au cours de son histoire, en particulier la contemporaine: l'économie et par conséquent dans les structures politiques, économiques et sociales, ainsi que dans les conceptions éthiques et gnoséologiques. Le phénomène processuel est considéré actuellement dans son insertion dans un monde qui, comme celui d'aujourd'hui, fait place, nécessairement et péniblement, à de nouvelles formes de vie en commun en abandonnant des particularités anachroniques et en tentant -peut-être la tentative extrême de l'humanité- de créer des instruments juridiques idoines pour surmonter le risque de la catastrophe immanente de notre époque thermonucléaire, en même temps fascinante et terrible. Enfin, la perspective constitutionnelle est celle d'une recherche anxieuse de valeurs sociales et individuelles de justice et de liberté ${ }^{19}$, valeurs nouvelles mais supérieures, recherche en somme d'un point d'ancrageau moins provisoire- dans le courant tempétueux des transformations actuelles.

Or, le progrès du Droit Processuel a fait que son analyse entre non seulement dans les domaines du Droit Constitutionnel; nous pouvons voir aujourd'hui le Droit Processuel traverser les frontières du droit national, et l'homme, dans le tourbillon de changements qu'il vit

17 Piero Calamandrei.- Proceso y Democracia; Op. cit.

18 Mauro Capelletti.- Proceso, Ideologias y Sociedad; Op. cit.

19 C'est pour cela que Procès et Justice sont des concepts étroitement liés, comme le sont justice et liberté. L'un n'existe qu'en fonction de l'autre et celui-ci ne pourrait se réaliser sinon a travers l'autre: Miglior giustizia attraverso maggior liberta. Piero Calamandrei- Processo e democrazia. Prefazione,CEDAM, Padova, 1954. 
actuellement, est déjà entré dans le domaine du Droit Processuel International ou Transnational.

Le Droit Processuel est seulement un, et seulement une la Théorie Générale du Procès, et partant de cette prémisse nous devons analyser comment le Droit Processuel a pris de l'importance qu'il a maintenant au sein du Droit International Public et Privé ; nous espérons que cette présence réussisse finalement à prendre part à l'harmonisation et à l'unification de certaines normes essentielles pour le développement d'un Droit Processuel Transnational.

Dans la ligne de ce qui est exposé précédemment, le Droit Processuel Pénal International mérite une mention à part puisque, en fin, il a réussi l'établissement de la Cour Pénale Internationale. Il est vrai que la nécessité pour la société de poursuivre d'une manière globale le jugement des nommés délits contre l'humanité a déterminé le développement plus dynamique de cette branche du Droit Processuel Transnational ; mais ceci ne peut ni ne doit signifier que dans le domaine du Droit Civil et Commercial nous perdions de vue l'important projet entrepris par l'American Law Institute et l'UNIDROIT avec la finalité d'obtenir dans quelque temps l'unification de certaines normes que nous estimons doivent être présentes dans toute législation processuelle nationale.

Aujourd'hui le Droit Processuel Civil nous invite non seulement à l'analyser à partir de la perspective des Droits Nationaux, sinon également de la perspective du Droit International Il suffit pour cela de voir qu'en Amérique Latine le Code Processuel Civil Type pour l'Amérique-Latine a acquis une telle présence que nos législations processuelles civiles actuelles ont essayé de suivre ce modèle de très près au profit de l'unification de la législation processuelle civile, en partant toujours de la prémisse qui dit que les institutions de Droit Processuel sont uniques et en conséquence peuvent être utilisées et recréées par les législations internes sans que cela signifie une modification de ses fondements.

\section{Genèse du projet}

Le Projet de Principes et Règles du Procès Civil Transnational date de 1995, lorsque l'American Law Institute entreprend la préparation 
de ce projet. Depuis l'année 2000 il compte sur l'appui de l'UNIDROIT qui a permis, entre autres importants aspects, d'atteindre un nombre important d'États qui font partie de cette organisme international. La conjonction des deux institutions a pour but de pouvoir diffuser ce projet dans différents Etats du Monde, et que son débat, son analyse et modifications permanentes, permettent d'obtenir une plus grande diffusion internationale de l'objectif principal du projet: la harmonisation et l'unification de la législation processuale civile et commerciale.

La question de rigueur à ce stade est la suivante : Pourquoi l'unification de la législation processuelle civile est-elle importante? La réponse, nous la trouvons tout d'abord dans un fait palpable pour nous tous : la dynamique actuelle des transactions civiles-commerciales rend nécessaire pour les opérateurs juridiques compter sur des principes de base et sur des règles générales pour obtenir la sécurité juridique que tout bailleur de fonds étranger exige comme condition indispensable, au préalable à tout débours patrimonial que son investissement requiert.

En Amérique Latine nous vivons aujourd'hui une époque où l'investissement étranger constitue la source de la plupart des revenus nationaux. Eh bien, l'un des obstacles que ledit investissement étranger trouve ici est la crainte d'affronter, dans un pays différent, avec une langue également différente, une réalité juridique qui peut être souvent très confuse pour le bailleur de fonds américain ou européen. Cette confusion est concrétisée dans l'infinité de normes qui règlent les fondements essentiels de la protection patrimoniale, en partant, naturellement des Constituions de chacun des États américains. En outre, si à la confusion et l'insécurité que cette diversité d'ordres juridiques peut provoquer, nous ajoutons une méfiance presque générale au sujet des activités des différents Pouvoirs Judiciaires de chacun des États latino-américains, et en plus, une insécurité également latente sur le plan de la législation processuelle qui règle les activités commerciales et civiles, nous obtiendrons un résultat immédiat: la diminution ou éradication de l'investissement étranger, dans des territoires où il est fondamental. Nous croyons qu'il est également pertinent de signaler que le développement récent des pays de l'Europe Orientale constitue une alternative certainement intéressante pour le bailleur de fonds américain, asiatique ou de l'Union Européenne; et si 
lesdits nouveaux États ont comme objectif d'assimiler rapidement les fondements essentiels d'un Etat de Droit, et la mise en place d'une Economie de Marché, où sont privilégiées les relations commerciales, la réponse de l'investisseur étranger sera de préférer lesdits États de l'Est européen au lieu de choisir l'Amérique Latine.

Voici l'un des fondements principaux du projet de Principes et Normes de la Procédure Civile Transnationale: prétendre uniquement que l'étranger qui se présente à un ordre juridique étranger puisse trouver une certaine sécurité dans le fait que toute controverse provenant de ses relations juridiques-économiques soit jugée conformément à des règles connues de lui-même. Celui-ci est peutêtre le plus important de tous les motifs pour lesquels l'Amérique Latine ne peut pas se permettre le luxe de se tenir à l'écart du Projet de Principes et Règles du Procès Civil Transnational.

Nous avons dit dans la première partie du présent rapport, que le Droit Processuel est seulement un, unitaire, et donc ses principes et institutions le sont également. Il faut reconnaître que cette unité du Droit Processuel est affectée par les usages, les coutumes et les antécédents juridiques que chaque système légal supporte depuis longtemps.

A titre d'exemple seulement, nous pourrions signaler que malgré nos antécédents et une langue commune et malgré l'intention d'uniformiser la Législation de la Procédure Civile latino-américaine, nous allons trouver des différences importantes dans le traitement processuel civil que reçoivent certaines institutions de ces pays.

La réalité sud-américaine est importante pour notre recherche car c'est la réalité selon laquelle se déroule notre activité universitaire et professionnelle, et parce que nous croyons qu'il faut promouvoir dans cette partie du continent une étude adéquate du projet mentionné.

Un point important qu'il faut analyser dans la Genèse du Projet de Principes et Règles du Procès Civil Transnational est la difficulté sous-jacente de la présence de deux systèmes légaux de base: le common law et le civil law. Cette coexistence au niveau international a fait que ce projet doive continuer selon les institutions et principes de chacun de ces systèmes, et que, finalement nous puissions sauver du Droit Processuel ces institutions élémentaires, c'est-à-dire, des institutions qui sont présentes dans les deux systèmes légaux et que nous pouvons désigner comme les concepts de base du droit processuel. 
Le second problème, croyons-nous, dans la genèse du projet, a été la traduction de ces principes et règles à des langues différentes, dans le but d'arriver à tous les bouts du monde. Pour illustrer et pour apprécier la difficulté de l'adéquation idiomatique requise pour cette tâche, nous pouvons signaler que dans le cas de la traduction à l'espagnol, on a eu besoin de plus d'un spécialiste pour adapter la traduction espagnole aux idiotismes et termes utilisés dans les différents pays latinoaméricains. En conséquence, nous croyons que l'inclusion au projet de membres, de conseillers et de chercheurs de plus d'un pays ibéroaméricain a été tres importante, car cela pourrait donner plus de richesse au contenu de la traduction car finalement ce que nous désirons obtenir est une traduction fiable des Principes et Règles, dans une version compréhensible et applicable dans tous les pays de langue espagnole où finalement l'on puisse avoir comme point de consensus élémentaire la définition des différentes institutions du droit processuel qui font partie du projet lui-même.

\section{Développement et évolution}

Au sujet du développement du Droit Processuel International en Amérique Latine il convient de citer Maekelt ${ }^{20}$ qui signale ce qui suit:

«Il faut tenir compte du domaine du Droit Procéssuel International, et tout particulièrement de la coopération judiciaire.

Les conventions approuvées dans toutes les conférences interaméricaines, sauf la cinquième, attestent cette affirmation: commissions rogatoires (1975) et leur protocole (1979); réception de preuves à l'étranger (1975) et leur protocole (1984); régime légal de pouvoirs pour être utilisés à l'étranger (1975); efficacité extraterritoriale des sentences et arbitrages étrangers (1979); accomplissement des mesures conservatoires (1979); preuves et informations sur le droit étranger (1979) et compétence dans la sphère internationale pour l'efficacité extraterritoriale des sentences étrangères (1984).

20 Tatiana Maekelt.- El Desarrollo del Derecho Internacional Privado en las Américas. En: http://www.oas.org/juridico/spanish/tatiana_maekelt.htm 
Les conventions appelées "processuelles" règlent presque tous les grands thèmes de Droit Processuel International. Il manquerait un domaine dont la codification se fait avec beaucoup de difficultés: celui qu'on appelle juridiction directe, c'est-à-dire compétence processuelle internationale. Obtenir l'accord des États membres est extrêmement difficile, c'est pourquoi ce thème n'a jamais été inclus dans la programmation des conférences spécialisées».

L'Amérique Latine est un continent jeune en voie de développement, si on le compare à l'Europe continentale qui cherche à s'unifier depuis longtemps. A ce propos il suffit de voir que depuis la naissance des républiques latinoaméricaines, il y a eu une tendance à la cohésion des différents pays indépendants. Le rêve de Bolivar de construire finalement une seule nation latino-américaine ne s'est pas réalisé, cependant cette pensée est toujours latente, et c'est pour cela qu'au début du XXIème siècle nous constatons que l'Amérique Latine n'est pas composée de pays totalement individuels, autrement dit, d'États craignant l'unification avec d'autres en certaines matières.

Des accords interaméricains tels que la propre création de l'Organisation des États américains, et d'autres comme l'ALENA, le Pacte Andin, le MERCOSUR, (qui malheureusement peut être affecté par la grave crise que vit aujourd'hui l'Argentine) ou l'ODECA (Organisation des États Centroaméricains) constituent les instruments internationaux qui concrétisent le désir latinoaméricain de s'unifier au moins en certains domaines.

Malheureusement, le nombre de microrégionalismes étatiques et culturels a empêché que soit créée une grande communauté des nations latino-américaines où l'on aurait comme objectif l'établissement d'un ordre juridique uniforme dans certains aspects. S'il est vrai que l'Organisation des Etats Américains a cherché une certaine unification de critères dans quelques aspects de la vie en société, de grands progrès n'ont pas été atteints pour l'unification, ce que nous pouvons apprécier dans la Communauté européenne. Ceci malgré le privilège que nous avons en Amérique Latine de parler une seule langue et d'avoir des ancêtres assez homogènes, avec des exceptions certaines, mais de toute façon une seule langue qui nous permet de voyager depuis le Cap Horn jusqu'au Mexique, sans perdre pour autant la possibilité d'utiliser la langue de Cervantes. De même, la coexistence des systèmes du Common 
Law et du Civil Law ne devrait pas être un grand obstacle pour l'unification de notre législation processuelle, puisque en Amérique latine ce dernier est prépondérant et fait partie de notre héritage colonial.

L'Amérique latine vit depuis les années 60 une rénovation dans le domaine du Droit Processuel. Historiquement, nos pays ont encore come système processuel de base la «Ley de Enjuiciamientos» espagnole (Loi de Mises en Accusations) de 1851. Au début du Siècle passé ont eu lieu deux modifications de la législation processuelle civile latinoaméricaine. Par exemple, le Pérou a promulgué en 1912 le Code de Procédures Civiles, en vigueur presque totalement jusqu'aux années 70 lorsque il a souffert une série de modifications dans le but de l'adapter aux nouvelles tendences qui étaient apparues au cours des décennies précédentes. Notre pays a essayé de modifier totalement sa législation processuelle à partir de 1940, sans avoir de succès jusqu'en 1992, lorsqu'un nouveau Code Processuel Civil est entré en vigueur. La législation processuelle civile péruvienne est le produit de nombreuses Commission Parlementaires, puisque durant les différentes étapes aussi bien démocratiques que dictatoriales on a essayé de conformer des commissions multisectorielles qui auraient pour objectif d'élaborer la législation processuelle Civile tant attendue. Finalement, après nombre de réussites et d'échecs, nous avons aujourd'hui une législation processuelle civile qui certes demande une modification urgente et des corrections, mais qui, pour une grande partie, a substitué le système ancien de procédure .

Dejà au XXIème siècle, lorsque les problèmes limitrophes-territoriaux entre les pays latinoaméricains ont pratiquement disparu, ou ils sont en voie de solution pour certains qui sont restés comme résidu du Siècle passé, c'est à l' Amérique Latine de réussir son unification.

Une partie du travail amorcé pour ladite unification, spécifiquement dans le domaine juridique, dont l'analyse est notre responsabilité, est développée par le Comité Juridique Interaméricain de la OEA, créé sur la base de ce que prévoit la Charte de l' Organisation des États Américains:

"Le Comité Juridique interaméricain Art. 99०

Le Comité Juridique Interaméricain a pour finalité de servir de corps consultatif de l'Organisation en affaires juridiques; promouvoir le 
développement progressif et la codification du droit international, et étudier les problèmes juridiques concernant l'intégration des pays en voie de développement du Continent et la possibilité d'uniformiser leurs législations dans tous les aspects considérés convenables."

Sur les bases de la norme internationale citée plus haut, le Comité Juridique Interaméricain a réalisé des conférences, des réunions et, en ce qui concerne la matière de notre recherche, il a conclu ce qui suit ${ }^{21}$ :

Intégration économique et libre commerce

Les processus d'intégration présentent, du point de vue juridique, des aspects qui doivent être l'objet d'une attention spéciale.

Le changement de la réalité internationale, comme résultat de l'ouverture et de l'internationalisation de l'économie, a créé la nécessité, pour celui qui participe dans l'échange de biens et services, de trouver des voies qui garantissent la sécurité juridique des transactions commerciales et dans les conflits qui pourraient surgir.

Dans le cadre mondial l'arbitrage commercial a démontré être un instrument idoine, agile et efficace pour fournir cette sécurité juridique et résoudre les controverses issues de l'activité commerciale.

Cependant, dans le cadre du continent américain, bien que les gouvernements aient fait des efforts pour être en accord avec cette nouvelle tendence internationale, et que dans ce but ils aient modernisé leurs législations et ratifié des conventions internationales qui réglementent l'arbitrage international, telles que les Conventions de New-York et Panama, l'utilisation de ce mécanisme comme moyen alternatif de solution de controverses a été précaire.

21 Rapport sur le déroulement des activites du Département de Coopération et diffusion juridique. Département de coopération et diffusion juridique sous-secrétariat d'affaires juridiques secrétariat général d'organisation des états américains washington d.c. avril 1999 : http://www.oas.org/defaultesp.htm 
Etant donnée cette nécessité, le Département de Coopération et Diffusion Juridique a fait des progrès dans le développement et la mise en place d'un programme de formation dans le but aider les pays dans l'étude, l'analyse et l'application pratique de ce mécanisme.

Formation et échange d'information et d'expériences sur l'arbitrage en Amérique latine

Ce projet a été réalisé conjointement avec la Commision Interaméricaine d'Arbitrage Commercial (CIAC) et ses Sections Nationales.

A cette occasion ont participé, comme conférenciers et tuteurs, 250 avocats distingués et des experts en arbitrage international. Les ateliers de formation ont eu lieu en Bolivie, Chili, Colombie, Equateur et Paraguay.

Ce programme a permis aux participants, grâce au développement des différentes étapes d'un arbitrage international, de se familiariser dans la gestion de ce mécanisme. Il a offert la possibilité de confronter, analyser et résoudre les affaires, aussi bien de procédure que les substantifs, qui apparaîssent dans un arbitrage international, et d'analyser les possibilités de leur développement dans les systèmes juridiques nationaux.

Ce projet a permis, en outre, de présenter un programme de diffusion de l'arbitrage international, dans chaque pays participant.

L'arbitrage comme moyen alternatif de solution de controverses et le développement juridique des amériques

L'importance de ce sujet et l'appui offert par les Centres de Conciliation et Arbitrage des Chambres de Commerce, la Commission Interaméricaine d'Arbitrage Commercial (CIAC), l'Association Ibéroaméricaine de Chambres de Commerce et l'Association Centroaméricaine de Chambres de Commerce, rendront possible la poursuite des activités de formation commencées l' année dernière.

Dans cette nouvelle phase, on comptera sur la participation de treize Etats membres, qui participeront au projet à travers les centres de conciliation et arbitrage des chambres de commerce. 
Ce projet représente un nouveau modèle de formation plus ample, par rapport à ce qui a été fait pour des cas précédents et se déroulera en partant de l'expérience accumulée par le Département en cette matière.

Il a été programmé la réalisation d'un atelier de formation régional en Amérique Centrale et sept ateliers additionnels dans plusieurs pays de lá région.

Ces ateliers permettront la formation aussi bien d'avocats que de professeurs sur les progrès les plus récents dans la matière. Ces ateliers serviront en outre de forum qui permettra de diffuser, entre les chefs d'entreprise et les commerçants, l'importance de l'arbitrage international comme méthode alternative pour la solution des conflits.

Dans la méthodologie employée dans ces ateliers, en outre les classes magistrales, sont utilisés des exercices pratiques de simulation qui promeuvent un apprentissage interactif qui s'est révélé plus efficace que les méthodes traditionelles d'enseignement.

La préparation du matériel employé pendant cet apprentissage et la préparation des ateliers facilitera aux Centres de Conciliation Arbitrage la réalisation périodique de cours de formation, avec des caractéristiques similaires à celles exposées ici. Avec cela, non seulement est assuré le maintien du projet mais également la possibilité d'élargir sa portée à cour et moyen terme.

Un composant important de ce projet est la formation qui sera donnée aux professeurs universitaires qui, nous l' espérons, avec l' appui des centres d' arbitrage et de conciliation des Chambres de Comerce de leurs pays respectifs, pourront travailler à la conception, formulation et incorporation de cours sur cette matière dans leurs centres d'enseignement respectifs. La participation de ces professionnels sera assurée par l'octroi de bourses, ce qui garantit une participation de choix et représentative des centres d'éducation supérieure.

Un autre élément innovateur de ce projet est la création, parmi les institutions participantes des différents pays, d' un réseau d'Échange d'Informations et d'Expériences. Ce réseau facilitera en outre la 
préparation et la réalisation des cours de formation périodique que le Projet se propose d'encourager, ainsi que la préparation et le contenu des cours dictés dans les universités, comme résultat de cet effort.

\section{Conférence sur solution de controverses sur le marché mondial}

Le Département de Coopération et Diffusion Juridique, la Commission Interaméricaine d'Arbitrage Commercial, conjointement avec la Section de Solution de Controverses de l'American Bar Association (ABA) et d'autres organisations, co-patronneront une conférence sur le sujet de la Solution de Controverses sur le Marché Mondial, qui s'est tenue du 2 au 4 avril 1998 à Washington D.C.

La conférence a compté sur l'assistance d'avocats de différents pays, qui se sont présentés pour examiner de différents aspects concernant les mécanismes de solution de controverses.

Parmi les sujets traités, se trouvent les avantages d'inclure des clauses d'arbitrage dans les contrats internationaux; l'importance des méthodes alternatives de solution de controverses comme une méthode effective pour réduire la congestion des cabinets judiciares; et les règles de la médiation entre différents groupes culturels».

La recherche et les efforts en faveur de l'unification de la codification pour le progrès des relations civilo-commerciales ne sont pas étrangères à l' Amérique Latine. Etant donné les caractéristiques spéciales des pays latino-américains, et en plus, les problèmes centraux qu'ils doivent affronter: situation de crise économique et recherche du respect des droits de l'homme, cette tâche d'unification en matière civil et commerciale est en partie en retard ${ }^{22}$.

«Dans une première étape, qui a précédé les mouvements européens d'unification juridique continentale, les pays de l'Amérique Latine se sont embarqués dans un projet ambitieux de codification du droit international privé. Une seconde étape plus pragmatique, dont les

22 Alejandro Garró.- "Armonización y Unificación del derecho privado en América Latina: esfuerzos, tendencias y realidades"; En: http://www.cnr.it 
protagonistes sont les CIDIP, a abandoné l'aspitation romantique de la codification globale et s'est concentré sur des sujets plus spécifiques. S'il est vrai que les efforts pionniers et compréhensifs d'unification du droit privé n'ont pas eu un grand succès, ceux qui ont été déployés par la CIDIP ont obtenu jusqu'à cette date l'approbation de 21 conventions interaméricaines. Les efforts en vue de la prochaine CIDIP, qui se tiendra à Mexique en 1994, confirment cette tendance unificatrice vers une codification progressive du droit international privé interaméricain.

Les travaux des CIDIPs ont été modestement limités en ce qui concerne la matière et l' espace. C' est ainsi que les sujets sur l' unification ou l'harmonisation ne se sont pas étendus au delà des questions concernant les critères de sélection du droit applicable et la coordination de mécanismes de coopération judiciare internationale. Même si l'on prévoit l'adhésion à ces conventions de pays qui n'appartiennent pas à la O.E.A., l'espace d'acceptation des instruments interaméricains n'excède pas dans la majorité des cas celui de l' Amérique Latine.

Cet objectif et ce qui a déjà été obtenu n'est pas dédaignable. Cependant, la réalité contemporaine du droit commercial international exige une reprogrammation au sujet de la convenance de conserver une position régionaliste dans les efforts d'unification du droit privé.»

Malgré ce qui est exposé ci-dessus, nous croyons que la recherche et les efforts en faveur de l' unification des normes en matière processuelle civile et commerciale doit continuer à faire partie de l'agenda du travail latinoaméricain, puisque nous avons un besoin urgent de compter sur des normes claires, des textes légaux non controvertis et des règles qui démontrent à la collectivité internationale que la sécurité juridique fait partie de la vie socio-économique latinoaméricaine.

Dans le contexte de ce qui est signalé précédemment, c'est encourageant de voir que le projet d'ALI-UNIDROIT sur les Principes et Règles du Procès Civil Transnational soit diffusé en Amérique Latine, puisqu' il constituera un encouragement de plus pour la recherche interne de l'unification de notre codification civile et patrimoniale, qui dans ce cas spécifique abordera les thèmes qui - ̀̀ cause du retard du Droit Processuel, auquel nous nous référons dans 
la première partie de ce Rapport- ne sont pas étudiés au jour le jour par les instituions ou organismes latino-américains qui encouragent l'unification.

\section{IV. État actuel}

L'état actuel du projet de Principes et Règles du Procès Civil Transnational en Amérique Latine est certainement encourageant puisque dans la révision du dernier Brouillon (Discussion Draft No 03) du 8 avril 2002, qui a été matière d'analyse et de discussion dans les réunions réalisées à Londres, Mexique et Roma, on a pu apprécier la plus grande collaboration d'experts latino-américains.

Nous considérons actuellement que ce Troisième Brouillon de discussion contient des concepts plus délimités, il y a un progrès remarquable dans sa rédaction et les définitions des instituions de Droit Processuel essaient d'être plus générales.

Malheureusement nous n'avons pas encore la traduction en espagnol de ce 3ème ProJet de Discussion, qui se fait aussi bien en Espagne que dans de différents pays latino-américains. Nous collaborons à cette traduction dans sa version destinée à l'Amérique du Sud.

Notre collaboration à ce Projet a consisté essentiellement à ce qui suit:

- Trouver la forme la plus adéquate de traduire les Principes et Normes à l'espagnol et réussir à ce que la traduction à l'espagnol contienne une terminologie adaptée à nos systèmes légaux.

- Réussir à unifier finalement une traduction en espagnol qui peut être utilisée dans tous les pays de langue espagnole.

- Analyser avec soin les incompatibilités des définitions, termes et institutions figurant dans le projet, et proposer une traduction en espagnol compatible avec le sens exposé dans sa version originale en anglais-.

- Apporter notre expérience professionnelle et universitaire en tant qu'opérateurs juridiques au texte qui finalement constituira la version définitive du projet, et,

- Diffuser l'étude et l'analyse du Projet par le biais des travaux de recherche de la Faculté de Droit de l' Université Catholique 
Pontificale du Pérou, et des pages de DERECHO-PUC, Revue Officielle de la Faculté de Droit de cet établissement universitaire. Il faut signaler à ce propos que nous avons déjà contribué à éditer le $2 \mathrm{e}$ Brouillon de Discussion, dans l' Édition No $52^{23}$ ainsi qu' au moyen de l' Edition d'un Tirage à part Spécial qui ne contient que le texte de ce Brouillon de discussion dans ses versions en espagnol et en portugais, et de plus, un important travail sur cette matière préparé par le Professeur Hazard ${ }^{24}$, coauteur des normes, ainsi que l' exposé du Prof. Antonio Gidi ${ }^{25}$, Rapporteur Associé dudit pojet.

Notre travail à l'UNIDROIT nous a permis d'accéder à une bibliographie précieuse dans le but de pouvoir élargir notre recherche sur les fondements essentiels de l'harmonisation et de l'uniformisation du procès civil, en prenant en considération que l'une des différences les plus importantes entre le Civil Law et le Common Law se réfere au système processuel que chacun de ces systèmes prévoit.

Pendant notre séjour à l'UNIDROIT à Rome, nous avons peut accéder à une information d'énorme importance pour la dynamique requise pour l'unification des deux systèmes processuels. En outre, pendant ce sèjour, comme les annès derniers a París, France, à New Orleans, Luisiana, et a Méxique, nous avons peut participer aux réunions de ALI-UNIDROIT à Londres et à Rome.

\section{Actions futures. Evaluation}

Comme nous l'avons déjà signalé, la traduction du 3e Brouillon de Discussion des Principes et Règles Transnationaux est en voie d'édition; cette étape de traduction ne pourra pas dépasser les trois mois prochains,

23 Édition Spéciale DERECHO PUC,; Fondo Ed. PUC du Pérou, Lima 1999

24 Geoffrey C. Hazard, J r..- Civil Litigation without frontiers: Harmonization and Unification of Procedural Law; (en anglais et espagnol) En: DERECHO PUC No 52, Revue de la Faculté de Droit de l'Université Catholique Pontificale du Pérou; Fondo Ed. PUC du Pérou. Lima 1999. Voir aussi: Édition Spéciale DERECHO PUC; Fondo Ed. PUC du Pérou, Lima 1999

25 Antonio Gidi.- A presentaçao ao Projeto às Normas Trasnacionais de Processo Civil; (en portugais et espagnol); Op. cit. 
du fait que l'agenda de ALI-UNIDROIT prévoit pour mai 2003 la réalisation de la prochaine Conférence d'évaluation du nouveau projet de discussion qui est en voie de réalisation par les auteurs du Projet Principes et Règles; et afin de pouvoir participer plus étroitement à ce projet, et réaliser correctement notre travail de collaborateurs étrangers au projet, nous avons besoin d'assister aux prochaines réunions sur la matière, mais surtout $\mathrm{d}$ ' avoir un texte qui nous serve de guide et de base pour suivre le cours des débats réalisés autour de ce projet.

Nous croyons important, par exemple, de souligner le travail que réalise l'Institut Mexicain de Droit Uniforme, qui a organisé la réunion tenue en février passé à Mexique D.F. Cette importante institution encourage la collaboration des chercheurs de langue espagnole et, grâce à son initiative nous sommes prêts à collaborer à l'oeuvre collective qui se prépare à ce sujet.

Pour notre part, nous croyons que notre travail de diffusion pourra s'enrichir avec l'organisation d' une réunion à Lima, Pérou, afin que le projet soit présenté officiellement, et pendant laquelle ses auteurs pourront exposer directement aux opérateurs juridiques péruviens quels sont les fondements, le développement et l' état actuel de l'élaboration de cet important projet.

Nous avons comme tâche en attente de sensibiliser l'État péruvien et ses autorités afin que finalement ils adhèrent à l'UNIDROIT, et ratifient son Statut. Nous avons déjà entrepris les démarches destinées à cette fin, et nous avons eu l' occasion de réunir à Lima le 14 juin de cette année un groupe important de juristes péruviens, à qui nous avons exposé l' importance de cette adhésion. Notre travail, dans ce sens, continue et nous avons reçu l'engagement des professeurs avec qui nous nous sommes réunis de le diffuser auprès des autorités nationales et solliciter- selon leurs possibilités- cette adhésion.

\section{Futur proche et mediat}

Le futur proche des Principes et Règles du Proces Civil Transnational s'encadre dans sa plus grande diffusion, avec la finalité de gagner des adeptes à cette noble cause, afin de pouvoir recueillir la plus grande quantité d'initiatives, d'appréciations, de commentaires, d'apports et de suggestions pour l'enrichir pour le bien de notre propre 
Communauté Internationale dans un futur proche, et obtenir ainsi que les opérateurs juridiques (chercheurs, avocats en exercice et membres de la magistrature) puissent connaître et manifester leurs appréciations et leurs critiques sur ce qui a été fait jusqu’à présent par les auteurs principaux du Projet, le Prof. G.C. Hazard et le Prof. M. Taruffo, et avec l' importante assistance du Prof. Antonio Gidi, Rapporteur Associé.

Nous sommes profondément convaincus que la diffusion de cet important Projet doit commencer dans les salles de classe des universités. Chaque collaborateur à ce Projet doit avoir comme but sa diffusion auprès de notre entourage universitaire, en essayant au moins d'attirer l'attetion de nos élèves de l'existence de ce Projet, pour promouvoir ensuite son étude dans chacune de nos Écoles de Droit, la recherche à son sujet et sa diffusion comme partie intégrante d'un cours qui enseigne les concepts élémentaires du Droit Processuel Transnational. C'est donc cette tâche même que nous devons entreprendre, nous, les professeurs universitaires, par le biais de l'enseignement du Droit Processuel et sa Théorie Scientifique, et j'insiste, nous ne devrions pas nous limiter au cadre local, mais être capables d'avancer d'un pas et de nous permettre de nous consacrer également à l'étude du Droit Processuel International, pour lequel ce Projet s'avère un effort avancé de spécialistes remarquables.

Comme conséquence des activités signalées ci-dessus, nous pouvons prédire que dans un futur proche le Projet de Principes et Règles pourra être accessible non seulement aux opérateurs juridiques actuels sinon et surtout aux futurs avocats, qui devraient au début de leurs activités professionnelles avoir en main cet instrument international de Droit Processuel International.

Le futur médiat de ce projet sera la mise en oeuvre adéquate adoptée par les États au sujet des principes et règles dans chacune de leurs législations. Nous pensons que cette tâche ardue ne va pas avoir un résultat immédiat puisque pour obtenir l'adéquation des principes et règles dans les différentes législations nationales il devra se passer beaucoup de temps, car une modification de telle nature demande non seulement le travail des chercheurs juridiques mais aussi la volonté politique des différents gouvernements d'adapter leurs législations à la nouvelle perspective du Droit Processuel Civil International. 